\title{
Attaining secure and sustainable water management for developing world
}

Keywords: anthropocene world, transport media, scientists, ecodiversity, earth, millennium development, environment, water

Abbreviations: MDGs, millennium development goals; SDGs, sustainable development goals; WS, water security

\section{Introduction}

From the stone-age era to the current Anthropocene world, water is central to our religious, scientific and technological civilizations. Water is used by many religious communities during baptisms supposedly to wash away spiritual sins. Scientists use water both as a solvent and transport media. However the best paradox is how nature utilizes water. In nature, water supports life systems such as growing food crops and as a media for elimination and transmission of diseases. When tamed, water presents a serene beauty and provides useful forms of energy to power human civilizations. However, when it becomes very unforgiving, hazards such as floods, Tsunamis, thunderstorms and landslides are some of the faces of water we witness. Likewise, lack of water can at times be as devastating from droughts and famines to dried water bodies that change both climate and eco-diversity. Some scholars argue that we may not need to build any new hospitals if we can manage our water resources well as water borne diseases kill more people per year than all categories of wars combined.

\section{Sustainability and security}

Human body just like that of the Earth is about 70 percent water. Thus, water is life but secured and sustainable water therefore becomes dignified and sustainable life. To enforce this important paradigm, UNESCO-IHP (2017), ${ }^{2}$ indicates that about $3 / 4$ of all new jobs worldwide are water related. The former prime minister of Norway, Brunt land, defined sustainability:

a. activities where the needs of the present generation are met without compromising the needs of future generations and

b. Equitable spatially and temporally distribution of the resources.

Although the central role that water occupies in sustainable development is recognized, water-related services remains far too low on the scales of public perception and of governmental priorities.

As a result, water which ordinarily should be a common good often becomes a limiting factor, rather than an enabler to social welfare, economic development and healthy ecosystems. According to United Nations (2015) score card report on Millennium Development Goals (MDGs), most of the indicators in attainment of Goal 7 were missed by many developing world countries. In 2016, the world embraced the Sustainable Development Goals (SDGs). In the SGDs, unlike in MDGs, the issues of water management were specifically itemized and prioritized in Goals 6 and 14 but with linkages to several other goals. The scientific community, through the Future Earth forum (Future Earth, 2017), intends not to miss the targets again as was the case with MDGs by collapsing the many SDGs into three broad areas

\author{
Volume I Issue 6 - 2017
}

Chrispin O Kowenje,' Andreas Haarstrick'
'Department of Chemistry, Maseno University, Kenya
${ }^{2}$ University of Braunschweig-Institute of Technology, Germany

Correspondence: Chrispin O Kowenje, Department of Chemistry, Maseno University, Kenya, Tel +2547I 0184204 Email ckowenje@maseno.ac.ke

Received: December 21, 2017 | Published: December 27, 2017

of: a) Dynamic planet (environment, water quality, etc.) for better understanding of drivers, interactions and processes in water issues, and anticipation of global thresholds and risks, b) Global sustainable development (water management, communications, awareness, etc.) for deeper understanding of ecosystem functions and services, and c) Transformations towards sustainability (waste management, ecopsychology, etc.) for sustainable integration of emerging technologies and economic development pathways in human development.

\section{Constraints on water resources management}

Besides increasing demands for water, there are additional constraints that pose significant challenges to improving water resources management. These transcend any type of pressure-stateresponse analysis, yet they are tangible and merit a critical level of consideration when addressing water-related issues in the context of sustainable development. ${ }^{3}$ Lack human and infrastructural capacity, Persistent poverty, discrimination and inequalities in access to drinking water and sanitation services, and insufficient and unsustainable financing for water resources management and services are some of the hindrances to sustainable water management. Additionally, according to exceed-swindon network (www.exceed-swindon.org) the following are the challenges to hindering the attainment of secure and sustainable water management in developing countries:

a. Water Pollution and treatment technologies,

b. Governance and sharing of water resource,

c. Impact of climate changes, and

d. Lack of and conflicting policies and data on water availability and accessibility.

By the same argument, there is need to determine the water security (WS) index of the above listed challenges for any meaningful development undertaking. Unlike the rest of the developing world, the East Asian continent has made germane attempts in integrating WS index in their development plans evaluations. Due its close connection with food security, water scarcity is often presented by both print and electronic media as food scarcity. Ideally, WS must of necessity include but not be limited to economic, agricultural, governance, environmental, water related disasters, and domestic water usage. 


\section{Way forward}

To secure and sustainably manage the water resources, multidisciplinarily in training is recommended. Arguably, such numerated multilayered challenges facing sustainable water management do not lend themselves to narrow disciplines. Broadly, the experts need to be able to identify pollutants and prevent of pollution and contaminations, advocate for good water resource governance, push for green revolution in afforestation and enhancement of carbon credits, develop early warning systems such as the utilization of modern (hydro-) meteorological station data, and integrate indigenous or traditional knowledge into early warning systems. Water security plays a pivotal role in human development and in attainment of the SDGs to an extent that of the 17 SDG goals about 9 are water related nexus. According to Crow-Miller et al. 2016, achieving water security in urban environments is important as cities already shelter more than half of the world's population and accordingly the highest concentration of socio-economic activities. In addition, the knowledge of renewable energy and clean alternative energy sources is encouraged. Primarily, collaboration among water management players and effective communication of scientific findings are key to offering sound, secure and sustainable water management systems.

\section{Acknowledgements}

None.

\section{Conflict of interest}

The authors declare that they have no conflict of interest.

\section{References}

1. Future Earth, Kenya.

2. Crow-Miller B, Chang H, Stoker P, et al. Facilitating collaborative urban water management through university-utility cooperation. Sustainable Cities and Society. 2016;27:475-483.

3. World Water Assessment Programme (WWAP), Kenya. 